\title{
Traveling-Wave Electroabsorption Modulated Laser Based on Identical Epitaxial Layer Scheme and HSQ planarization
}

\author{
Ali Al-Moathin ${ }^{1}$, Chong Li ${ }^{1}$, Jue Wang ${ }^{1}$, Qusay Raghib Ali Al-Taai ${ }^{1}$, Iain Eddie ${ }^{2}$, Shengwei Ye ${ }^{1}$, \\ Lianping Hou ${ }^{1}$, Stephen Thoms ${ }^{1}$, Anthony Kelly ${ }^{1}$, and John H. Marsh ${ }^{1}$ \\ 1. James Watt School of Engineering, University of Glasgow, Glasgow G12 8QQ, U.K. \\ 2. Sivers Photonics Ltd., Glasgow G72 OBN, U.K.
}

Electroabsorption modulated lasers (EMLs), comprising a distributed feedback (DFB) laser and electroabsorption modulator (EAM) monolithically integrated into the same chip, are attractive because of their compact size, low fabrication cost, and their capability to offer a high modulation speed with low drive voltage, low chirp, and high extinction ratio [1,2]. The modulation speed of the EML is limited by the $R C$ constant of the EAM electrode, which is conventionally configured with either a lumped or travelling-wave (TW) electrode. The latter approach overcomes the $R C$ limit by including the EAM in a microwave circuit matched to the source [3]. However, due to restrictions imposed by size and materials, TW EAMs have to date been integrated externally using a specifically designed material structure or monolithically using selective area growth.

In this work we report, for the first time, an EML integrated with TW EAM based on the identical epitaxial layer design and using a side-wall grating (SWG) for the DFB laser. A novel planarization method based on hydrogen silsesquioxane (HSQ) spin-on coating [4], was used to create a 2.4- $\mu$ m-thick low-permittivity film $\left(\varepsilon_{r} \approx\right.$ 3.8) on which the TW electrode was fabricated. The EML chip comprised a DFB, EAM, and semiconductor optical amplifier (SOA) of lengths $600 \mu \mathrm{m}, 150 \mu \mathrm{m}$, and $320 \mu \mathrm{m}$, respectively. The devices were initially integrated with a single common $2.5-\mu \mathrm{m}$-wide ridge separated by $30-\mu \mathrm{m}$-long electrical isolation sections. The EAM section was deep etched to reduce the $p-i-n$ junction capacitance. In the DFB section, the ridge waveguide was fabricated with a SWG of 0.6- $\mu$ m-recess-depth, a Bragg grating period of $246 \mathrm{~nm}$ and included a $\lambda / 4$ phase shift at the centre of the DFB cavity. Images of the shallow-, deep-etch, isolation, and grating sections are shown in Fig. 1(a) and (b), and the final fabricated EML is shown in Fig. 1(c).
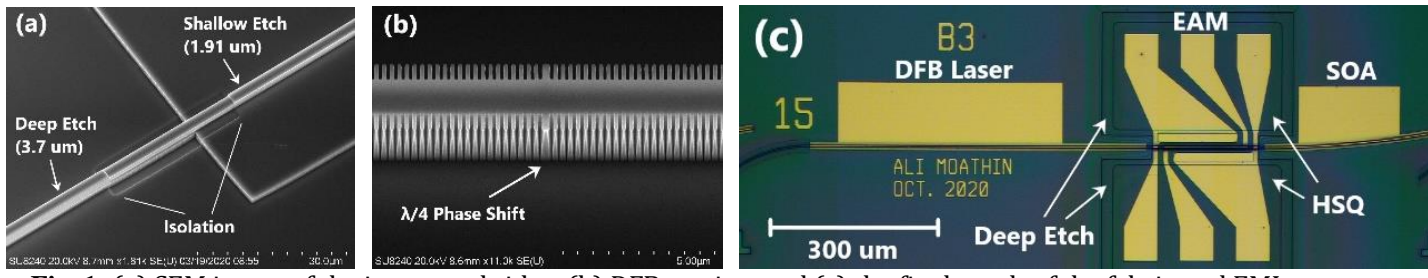

Fig. 1. (a) SEM image of the integrated ridge, (b) DFB grating, and (c) the final result of the fabricated EML.

The lasing wavelength was $1565 \mathrm{~nm}$ at a drive current of $60 \mathrm{~mA}$ and the side mode suppression ratio was $>35 \mathrm{~dB}$. The device exhibited stable single mode operation with no mode hopping. The extinction ratio of the EAM reached $22 \mathrm{~dB}$ at a bias voltage of $-4 \mathrm{~V}$. One-port Sparameter measurements were made on the coplanar waveguide (CPW) input port (bottom) of the EAM with the other port (top) terminated with a $50 \Omega$ load. Fig. 2 show the measured $S_{11}$ and equivalent circuit of the EAM. The results indicate a poor port match except around 39 $\mathrm{GHz}$ and therefore an appropriate matching circuit is required to improve the device RF performance.

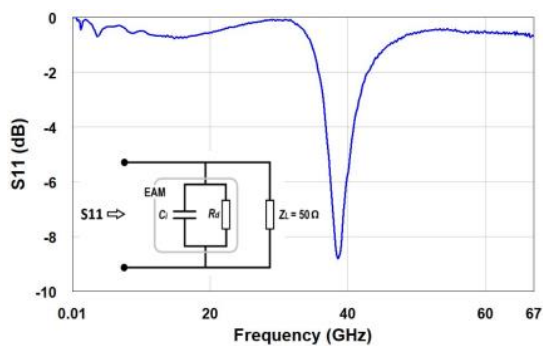

Fig. 2. $S_{11}$ parameter.

In summary, a new EML has been fabricated using a simple and low-cost process. The planarization method provides a route to manufacturing using regrowth-free fabrication and incorporating transmission line technology. The electrical results suggest the device can provide a high modulation speed with low drive voltage, stable operation, and a high extinction ratio. However, the port measurements of the TW electrode indicate an appropriate matching is required to achieve the highest possible modulation speeds.

\section{References}

[1] M. Theurer, G. Przyrembel, A. Sigmund, W. D. Molzow, U. Troppenz, and M. Möhrle, "56 Gb/s L-band InGaAlAs ridge waveguide electroabsorption modulated laser with integrated SOA," Phys. status solidi, vol. 213, no. 4, pp. 970-974, 2016.

[2] T. Y. and F. K. W. Kobayashi, M. Arai, N. Fujiwara, T. Fujisawa, T. Tadokoro, K. Tsuzuki, "Wide temperature range operation of 10-

/40-Gbps 1.55- $\mu \mathrm{m}$ electroabsorption modulator integrated DFB laser,” OECC2010 15th, vol. 1, no. July, pp. 9-10, 2010.

[3] S. Jeon, Y. Chung, Y. Kang, and J. Kim, "Modeling of Traveling Wave Electro-absorption Modulator for High Speed Optical Communication Systems," 2005 IEEE Conf., pp. 467-470, 2005.

[4] A. Al-Moathin et al., "Thick film hydrogen silsesquioxane planarization for passive component technology associated with electronicphotonic integrated circuits," J. Vac. Sci. Technol. B, Nanotechnol. Microelectron., vol. 37, no. 6, p. 61210, 2019. 